\title{
Evaluation of Anatomical Variations in Root and Canal Morphology of Primary Maxillary Second Molars: A Cone- beam Computed Tomography Study
}

\author{
Navin H Krishnamurthy ${ }^{1}$, Sharon Jose ${ }^{2}$, Umapathy Thimmegowda ${ }^{3}$, Prasanna K Bhat ${ }^{4}$
}

\begin{abstract}
Introduction:Visualizing the pulp cavity requires adequate knowledge of the size, morphology, and variation of the root canals of primary teeth. The morphology of the root canals in deciduous teeth causes difficulties during endodontic treatment. There have not been many studies on root canal variations in primary teeth in the Indian population.

Aim and objective: To assess the variation in the root and canal morphology of primary maxillary second molars using cone-beam computed tomography (CBCT) in the pediatric Indian population.

Materials and methods: The institutional database and private diagnostic centers were used to gather CBCT images of 25 children (50 images) between the age-group of 5 years and 9 years. The CBCT images were rebuilt using Scanora software, and the data were assessed and analyzed using the SPSS version for Windows.

Results: Out of 50 primary maxillary second molars, the majority of the second molars had three roots and three canals (Variant I) which account for $66 \%$ of teeth. Variant II had three roots and three canals with distobuccal and palatal roots fused in $14 \%$ of cases whereas $18 \%$ of cases showed three roots and four canals in which mesiobuccal root had two canals (Variant III). The remaining $2 \%$ of cases showed four roots with four canals (Variant IV) which were statistically significant.

Conclusion: We concluded that within the constraints of our study, there was a difference in the root canal configuration of primary maxillary second molars in the investigated group of the Indian population.
\end{abstract}

Keywords: Cone-beam computed tomography, Deciduous molars, Root canal morphology.

International Journal of Clinical Pediatric Dentistry (2021): 10.5005/jp-journals-10005-2030

\section{INTRODUCTION}

Caries is the most common etiological factor that leads to pulpal involvement, which necessitates treatment to preserve the integrity of dental tissues. ${ }^{1}$ Primary teeth have a thinner layer of mineralized tissue between the external and interior surfaces of teeth than permanent teeth, allowing bacteria to readily penetrate the pulp. ${ }^{2}$

Changes in the chronology and eruption sequence of permanent teeth are one of the major problems produced by the premature loss of primary teeth; thus, conserving primary teeth in children is an essential concept that commonly requires endodontic treatment. ${ }^{3}$ Because of the unique internal geometry of the pulp cavity and features not frequently found in permanent teeth, such as connections involving furcation and horizontal anastomosis root canal therapy in primary teeth is regarded as exceedingly complex. ${ }^{2,4}$ Endodontic therapy requires a thorough understanding of the shape and diversity of the root canals of primary teeth. ${ }^{4}$ Aside from that, as root resorption begins, dentin is deposited within the root canal system, which can drastically alter the size, shape, and number of root canals. ${ }^{1}$

Dental periapical radiographs are commonly used for diagnostic purposes and but, such radiography provides only twodimensional images of a three-dimensional structure, potentially resulting in treatment plan errrors. ${ }^{5}$

Cone-beam volumetric tomography (CBVT) or cone-beam computed tomography is an efficient tool that provides precise pictures of the dentoalveolar areas for disease detection and is even useful in the field of endodontics. ${ }^{6}$ It has a less radiation dose than medical CT and higher resolution than conventional radiography
${ }^{1-4}$ Department of Pedodontics and Preventive Dentistry, RajaRajeswari Dental College and Hospital, Bengaluru, Karnataka, India

Corresponding Author: Navin H Krishnamurthy, Department of Pedodontics and Preventive Dentistry, RajaRajeswari Dental College and Hospital, Bengaluru, Karnataka, India, Phone: +91 9845272314, e-mail: navina30@yahoo.co.in

How to cite this article: Krishnamurthy $\mathrm{NH}$, Jose $\mathrm{S}$, Thimmegowda U, et al. Evaluation of Anatomical Variations in Root and Canal Morphology of Primary Maxillary Second Molars: A Cone-beam Computed Tomography Study. Int J Clin Pediatr Dent 2021;14(5):628-632.

Source of support: Department of Pedodontics and Preventive Dentistry, RajaRajeswari Dental College and Hospital, Mysore Road, Bengaluru.

Conflict of interest: None

technique. When utilized with a limited field of view (FOV) and small voxel size, it is currently regarded as the "GOLD standard" for determining root canal anatomy. ${ }^{5,7}$ It is also possible to obtain segments from various levels that do not contain projection errors due to superimposition and magnification, allowing for more precise three-dimensional views of the teeth. ${ }^{5}$

A large number of studies have been carried out in permanent teeth regarding various root canal configuration/classifications which have been proposed, and well documented in the literature. However, studies using CBCT to detect root canal morphology of primary teeth in Indian children are quite uncommon. ${ }^{2}$ 
Hence, the purpose of this study was to determine the number and morphology of root canals in primary maxillary second molars, as well as the applicability of CBCT in determining root canal morphology.

\section{Materials and Methods}

This study was carried out on 50 CBCT images of primary maxillary second molars of children aged between 5 years and 9 years to assure the original form of the morphology of the root canals. The images were collected from the institutional database and other private diagnostic centers, that were taken for various diagnostic purposes. The teeth which require pulp therapy with or without periapical lesions, minimal root resorption was included in the study, and teeth involving multi-surface carious lesion, teeth with more than half length of root resorbed, fractured teeth, and teeth with less bone coverage were excluded.

The ethical clearance was obtained before the commencement of the study from the institutional ethics and research committee.

\section{Radiographic Techniques}

A SCANORA $^{\oplus}$ 3D scanner was used to capture CBCT images at $90 \mathrm{kV}$ and $12.5 \mathrm{~mA}$, with a field of view of $60 \mathrm{~mm}$ and a voxel size of $0.1 \mathrm{~mm}$. The exposure length was 15 seconds and the slice thickness was limited to a minimum of roughly $1 \mathrm{~mm}$. Scans were performed by a certified radiologist using the manufacturer's specified protocol, and all images were taken with the least exposure, radiation dose, and field required for optimal picture quality.

\section{Evaluation of the Image}

With the help of ON DEMAND 3D and Scanora software, the 50 $\mathrm{CBCT}$ images were reconstructed in coronal slices and evaluated. To ensure optimal viewing, the software's image processing function was used to modify the contrast and brightness of the image. The number of roots, number of canals per root, and root canal variations were all assessed. The analyzing criteria were based on those proposed by Gozde Ozcan in 2015 (Fig. 1).

\section{The Four Variants Which Were Analyzed in this Study}

Variant I: Three separate roots with each root having one canal.

Variant II: Three roots with three canals where distobuccal and palatal roots are fused.

Variant III: Three roots with four canals (mesial-2, distal-1, palatal-1).

Variant IV: Four roots with four canals (mesiobuccal, mesiopalatal, distobuccal, distopalatal) (Fig. 2).

Variant I, II, and IV correspond to class I, class III, and class IV in Gozde Oscan's root canal variations. Variant III was not mentioned which distinguishes this study from his root canal variations.

\section{Statistical Analysis}

SPSS version 22.0 (IBM, Corp.) for Windows was used to analyze the study data. The Chi-square test was used to compare the frequency of the number of root and canals, with a significance level of $p<0.05$.

\section{Results}

Fifty $C B C T$ images of primary maxillary second molars were assessed. The average age of the studied images was 6.8 years for males $(n=15)$ and 7.6 years for females $(n=10)$. The preponderance of primary maxillary second molars had three roots separately with each root having one canal (Variant I) which account for $66 \%$, Variant II had three roots and three canals with the distobuccal and palatal root are fused shown in $14 \%$ teeth and $18 \%$ had three roots and four canals where mesiobuccal root had two canals (Variant III). The remaining $2 \%$ showed four roots with four canals (Variant IV) which were statistically significant (Table 1).

The Chi-square test revealed that root and canal morphology of maxillary second molar teeth did not differ significantly according to gender.

The gender-wise distribution of primary maxillary second molar revealed that $90 \%$ of Variant I were seen in males whereas only $50 \%$ were seen in females. Variant II, teeth with distobuccal and palatal canal fused were seen in $25 \%$ of females but, none of them were seen in males. While, Variant III was found in $10 \%$ of males and $25 \%$ of females, respectively (Table 2 ).

The four variants in root canal morphology were identified in primary maxillary second molars (Figs 3 to 6].

Apart from the four classes of root canal morphology of maxillary molar by Godze Ozcan, we have noticed a variant, i.e., Variant III-three root with four canals where mesiobuccal root had two canals which was not much reported in the literature (Fig. 7).

\section{Discussion}

The main aim of endodontic treatment for primary teeth is to avoid premature loss of due to carious exposure. ${ }^{3}$ Maintaining primary teeth in the dental arch is critical for proper tooth spacing, mastication, vocalization, esthetics, and the prevention of psychological repercussions from tooth loss. ${ }^{8}$ As root canal treatment in primary teeth is a significant challenge for pediatric dentists due to variations in root canal anatomy, a thorough understanding of the root and canal morphology of deciduous teeth can considerably increase treatment effectiveness. ${ }^{9}$

In the current study, CBCT was used to assess the number of root and canal morphology in 50 primary maxillary second molars of 25 individuals. Anatomically, the primary maxillary second molar is comparable to the permanent maxillary first molar; however, it is smaller in size. The mesiobuccal pulp horn is the largest and closest

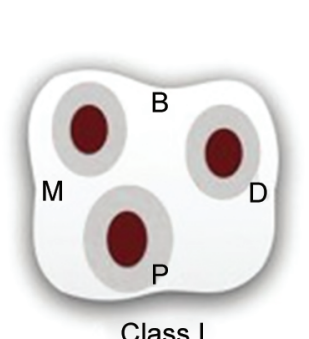

Class I

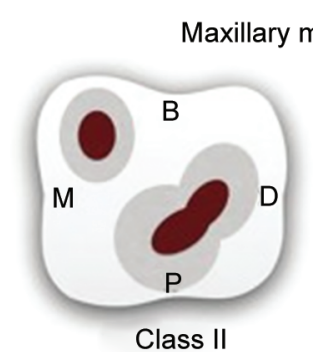

Class II
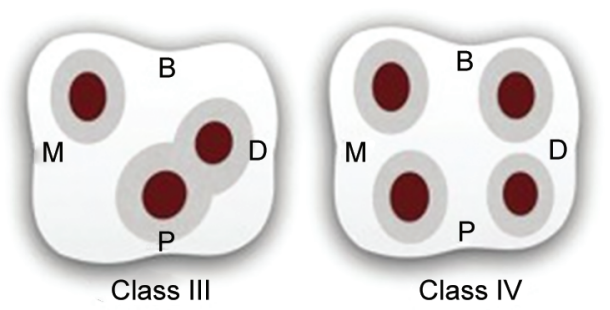

Fig. 1: Images of evaluation 


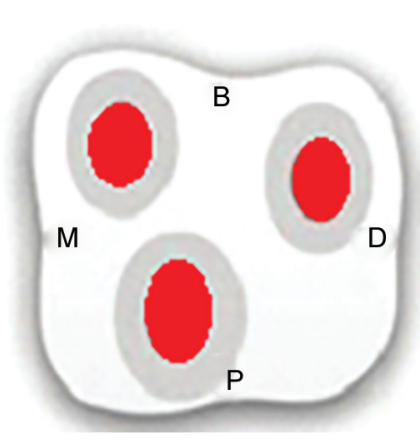

Variant I

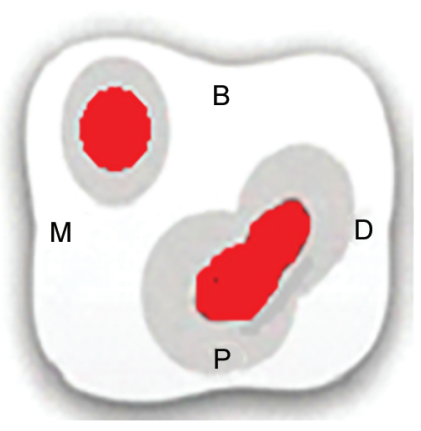

Variant II

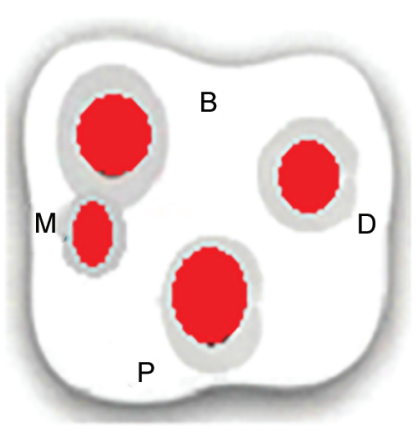

Variant III

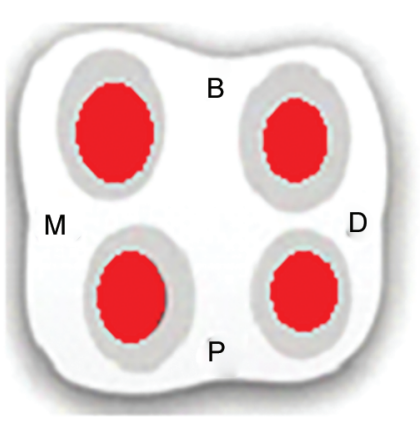

Variant IV

Fig. 2: Variants in root canal morphology of primary maxillary second molar

Table 1: Gender-wise distribution of root canal variants analyzed in the maxillary second molar teeth

\begin{tabular}{|c|c|c|c|c|c|c|c|c|}
\hline \multirow[b]{2}{*}{ Gender } & \multicolumn{2}{|c|}{ Variant I } & \multicolumn{2}{|c|}{ Variant II } & \multicolumn{2}{|c|}{ Variant III } & \multicolumn{2}{|c|}{ Variant IV } \\
\hline & $n$ & (\%) & $N$ & (\%) & $n$ & (\%) & $N$ & (\%) \\
\hline Males & 23 & 69.7 & 2 & 28.6 & 4 & 44.4 & 1 & 100 \\
\hline Females & 10 & 30.3 & 5 & 71.4 & 5 & 55.6 & 0 & 0 \\
\hline Total & 33 & 66.0 & 7 & 14.0 & 9 & 18.0 & 1 & 2.0 \\
\hline$\chi^{2}$ value & 4.561 & & 5.227 & & 0.128 & & - & \\
\hline$p$ value & 0.03 & & 0.02 & & 0.87 & & - & \\
\hline
\end{tabular}

Table 2: Gender-wise comparison of bilaterally symmetrical root and canal variants for maxillary II molars

\begin{tabular}{|c|c|c|c|c|c|c|c|}
\hline \multirow[b]{2}{*}{ Teeth } & \multirow[b]{2}{*}{ Variants } & \multicolumn{2}{|c|}{ Males } & \multicolumn{2}{|c|}{ Females } & \multirow[b]{2}{*}{$\chi^{2}$ value } & \multirow[b]{2}{*}{$p$ value } \\
\hline & & $n$ & $(\%)$ & $n$ & $(\%)$ & & \\
\hline \multirow[t]{3}{*}{ Max. II Molars } & Variant I & 9 & 90.0 & 4 & 50.0 & 4.085 & 0.13 \\
\hline & Variant II & 0 & 0.0 & 2 & 25.0 & & \\
\hline & Variant III & 1 & 10.0 & 2 & 25.0 & & \\
\hline
\end{tabular}

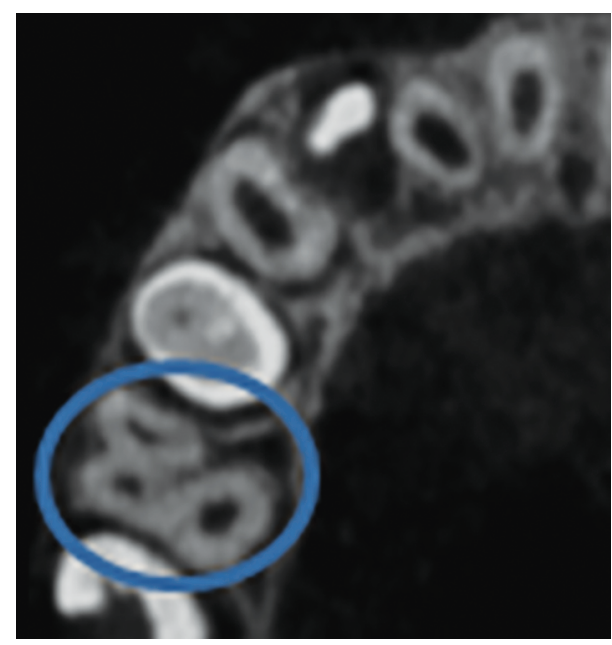

Fig. 3: Variant I: Three separate roots with each root having one canal

to the dentino-enamel junction. ${ }^{10}$ Yin-Lin et al. conducted an in vitro study on extracted primary molars (29 teeth), including maxillary and mandibular first and second molars, and found that the number of roots and root canals varied from two to three and three to four in primary molars. Although there were more one-canal roots in maxillary molars than two-canal roots in mandibular molars, there were equal numbers in both. In maxillary molars, a fusion of the distobuccal and palatal roots was prevalent. ${ }^{11}$ Rajendran et al.

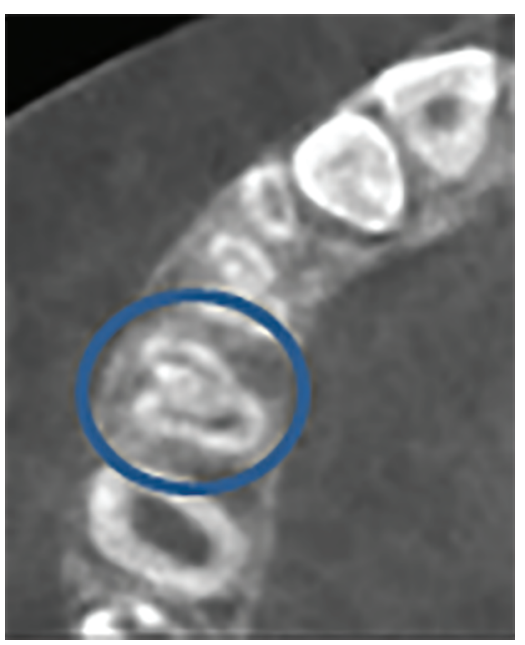

Fig. 4: Variant II: Three roots with three canals but distal and palatal root fused

conducted an in vitro study on 30 extracted primary maxillary molars using a spiral computed tomography scan, which revealed that two of the samples had two canals, with the distobuccal and palatal root canals fused, and 13 samples had three canals. Two samples of primary maxillary second molars had four canals (mesiobuccal, distobuccal, and two palatal), which is consistent with our findings in this study. ${ }^{9}$ Fumes et al. used micro-computed 


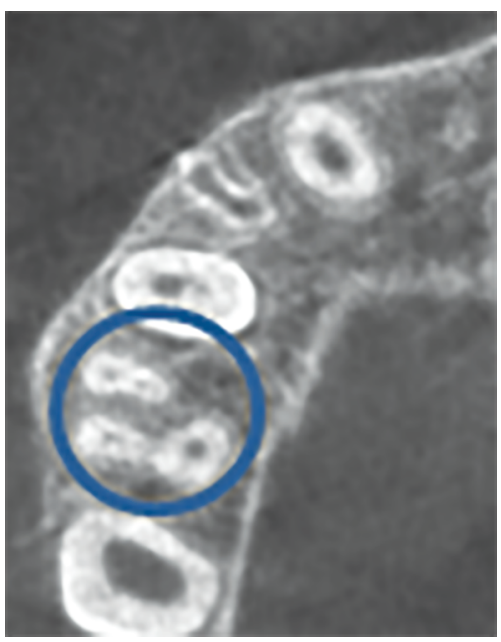

Fig. 5: Variant III: Three roots with 4 canals (mesial -2 , distal-1, palatal -1)

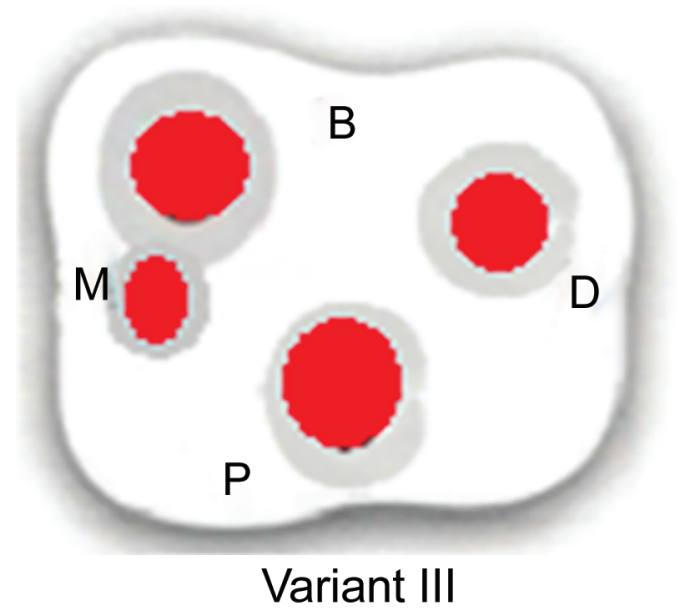

Fig. 7: Variant III: Three roots with four canals

tomography to analyze the root canal morphology of primary molar teeth, finding a double canal system in the mesial root of mandibular molars and mesiobuccal roots of maxillary molars which are consistent with our findings with mesiobuccal roots. ${ }^{3}$

An in vitro study conducted by Zoremchhingi et al. utilized computerized tomography to analyze the morphological variations in the root canal of 60 deciduous molars and found that the mesiobuccal root had one canal in $46.6 \%$ of the teeth and two canals in $53.3 \%$ of the teeth in an in vitro analysis. In the case of distobuccal roots, however, one canal was found in $73.3 \%$ of teeth and two canals in $26.6 \%$ of teeth. Similar canal variations were also found in the palatal canal. In the maxillary second molar, there were no combined distobuccal and palatal roots, which contrasted with our study, in which $14 \%$ of the distobuccal and palatal roots were fused. ${ }^{12}$

However, in this CBCT study, four root canal variations were observed. Variant I was three separate roots with one canal each which was most commonly observed and account for $66 \%$, Variant II was three roots with three canals (14\%) where the distobuccal and palatal root were fused, Variant III was noticed in $18 \%$ of teeth which had three roots with four canals where mesial root had two canals while distal and palatal root with one canal each. Variant IV was

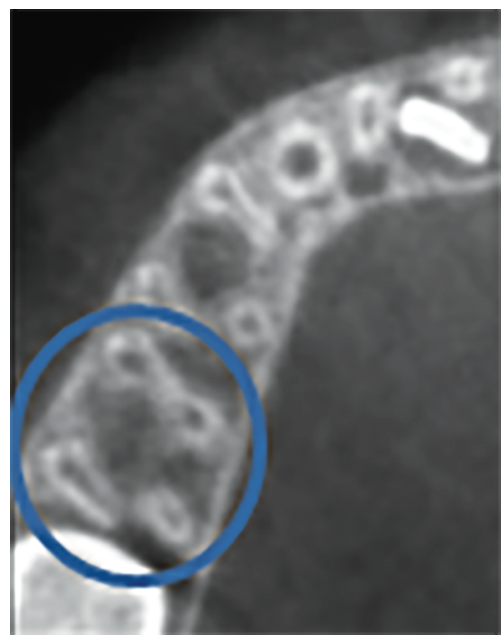

Fig. 6: Variant IV: Four roots with 4 canals

four roots with four canals (mesiobuccal, distobuccal, mesiopalatal, and distopalatal roots with one canal each) which were very rare.

In the present study, CBCT was found to be an effective and accurate diagnostic tool for investigating root canal anatomy than conventional radiographs, because it enables visualization of fine details in three-dimensional imaging without visual noise, also allows the overlapping visualization of adjacent structure and even assure the patients with the lowest exposure rate in radiation. ${ }^{6}$ Our study reveals information on the variations of root canal morphology that helps in the success of pulp therapy in primary maxillary molar teeth.

Till today, no classification regarding root canal morphology of primary molars has been proposed unlike that of permanent teeth. ${ }^{13}$ However, Godze Oscan has found four classes (class I-IV) in the root canal of primary molars. ${ }^{14,15}$ But in our study, apart from the four classes (class I-IV), we found one more variant which has not been much reported in the literature. As a result, this research suggests that $C B C T$ is useful in evaluating the root and canal system, but it should not be utilized routinely. Only when traditional radiography techniques are unable to provide sufficient data for effective diagnosis and treatment planning, CBCT can be effectively used.

\section{Limitation}

The small sample size of this study was a limitation. As a result, more research is needed to find a greater number of variants that can aid pediatric dentists in increasing their diagnostic and endodontic treatment abilities.

\section{Conclusion}

This in vitro investigation revealed the different root canal variations in primary maxillary second molars, which is important for both diagnostic and pulpal therapy success in children. Most of the primary maxillary second molars have three roots with three separate canals, and the variety of root canal variants should be taken into account while undergoing endodontic therapy. When compared with traditional radiography, CBCT delivers precise and effective information concerning differences in root canal morphology in a three-dimensional image. This could allow clinicians for the development of appropriate management 
strategies to address the issue encountered during endodontic therapy in these teeth.

\section{Importance of the Present Study}

- We analyzed a variant, i.e., Variant III-three roots with four canals where the mesiobuccal root had two canals, which was not well described in the literature, in addition to the four classes of root canal morphology of primary maxillary molars proposed by Godze Ozcan.

- $\quad \mathrm{CBCT}$ is a useful method for precisely examining the root and canal system of primary teeth, but it should be used with caution.

- The majority of primary maxillary second molars have three roots with three canals, and the variety of root canal variants should be taken into account while performing clinical treatments, as it can improve the effectiveness in the pulp therapy of the primary teeth.

\section{Acknowledgments}

We thank Dr Balaji, Professor and Head, Department of Oral Medicine and Radiology, RajaRajeswari Dental College and Hospital, for his constant support without which this study would not have been possible.

\section{References}

1. Reddy NV, Daneswari V, Patil R, et al. Three-dimensional assessment of root canal morphology of human deciduous molars using cone beam computed tomography: an in vitro study. Ind J Pedod Rehabil 2018;3(1):36-41. DOI: 10.4103/ijpr.ijpr_21_17.

2. Yang R, Yang C, Liu Y, et al. Evaluate root and canal morphology of primary mandibular second molars in Chinese individuals by using cone-beam computed tomography. J Formos Med Assoc 2013;112(7):390-395. DOI: 10.1016/j.jfma.2012.10.008.

3. Fumes AC, Sousa-Neto MD, Leoni GB, et al. Root canal morphology of primary molars: a micro-computed tomography study. Eur Arch Paediatr Dent 2014;15(5):317-326. DOI: 10.1007/s40368-014-0117-0.
4. Ozcan G, Sekerci AE, Cantekin K, et al. Evaluation of root canal morphology of human primary molars by using CBCT and comprehensive review of the literature. Acta Odontol Scand 2016;74(4):250-258. DOI: 10.3109/00016357.2015.1104721.

5. Acar B, Kamburoğlu K, Tatar I, et al. Comparison of microcomputerized tomography and cone-beam computerized tomography in the detection of accessory canals in primary molars. Imag Sci Dent 2015;45(4):205-211. DOI: 10.5624/isd.2015.45.4.205.

6. Rouhani A, Bagherpour A, Akbari M, et al. Cone-beam computed tomography evaluation of maxillary first and second molars in Iranian population: a morphological study. Iran Endod J 2014;9(3):190.

7. Przesmycka A, Tomczyk J. Differentiation of root canal morphology-a review of the literature. Anthropol review 2016;79(3):221-239. DOI: 10.1515/anre-2016-0018.

8. Bagherian A, Kalhori KA, Sadeghi M, et al. An in vitro study of root and canal morphology of human deciduous molars in an Iranian population. J Oral Sci 2010;52(3):397-403. DOI: 10.2334/ josnusd.52.397.

9. Vijayakumar R, Selvakumar H, Swaminathan K, et al. Root canal morphology of human primary maxillary molars in Indian population using spiral computed tomography scan: an in vitro study. SRM J Res in Dent Sci 2013;4(4):139. DOI: 10.4103/0976-433X.125587.

10. Mathewson RJ, Primosch. RE. Fundamentals of pediatric dentistry. 3rd ed., Quintessence Publishing Co, Inc, pp. 197-205.

11. Wang $\mathrm{YL}$, Chang $\mathrm{HH}$, Kuo $\mathrm{Cl}$, et al. A study on the root canal morphology of primary molars by high-resolution computed tomography. J Dent Sci 2013;8(3):321-327. DOI: 10.1016/j.jds.2013. 04.002 .

12. Joseph T, Varma B, Mungara J. A study of root canal morphology of human primary molars using computerised tomography: an in vitro study. J Indian Soc Pedod Prev Dent 2005;23(1):7. DOI: 10.4103/09704388.16019.

13. Salama S, Carole H, Davis. M. Anatomy of primary incisor and molar root canal. Pediatr Dent 1992. 14.

14. Baccouche C, Ghoul-Mazgar S, Baaziz A, et al. Topography of the pulp chamber in the maxillary primary molars of Tunisian children. Indian J Dent Res 2013;24(2):206. DOI: 10.4103/0970-9290.116682.

15. Gaurav V, Srivastava N, Rana V, et al. A study of root canal morphology of human primary incisors and molars using cone beam computerized tomography: an in vitro study. J Indian Soc Pedod Prev Dent 2013;31(4):254. DOI: 10.4103/0970-4388.121827. 